ARTÍCULO ORIGINAL

\title{
Sacroileítis: evaluación por resonancia magnética según criterios ASAS
}

\section{Sacroilititis: diagnosis for magnetic resonance according to ASAS criteria}

\author{
Silvia L. Espinoza-Alvarado*, Mariano Mejico, Juan M. Montero, Rodrigo del Cerro y Fabrizzio Galiano
}

Servicio de Diagnóstico por Imágenes, Diagnóstico Médico Oroño, Rosario, Santa Fé, República de Argentina

\section{RESUMEN}

Antecedentes: La resonancia magnética (RM) se ha convertido en la modalidad de imagen más relevante para el diagnóstico y la clasificación de la espondiloartritis axial temprana, incluida la espondilitis anquilosante temprana. Objetivos: Determinar la prevalencia de sacroileítis, identificar y describir los hallazgos de RM utilizando los criterios de clasificación ASAS (Assessment of SpondyloArthritis Internacional Society). Método: Un total de 80 pacientes con sospecha clínica de sacroileítis e indicación de RM sacroilíaca se sometieron a RM sin gadolinio en un resonador de 1.5 Tesla. Se realizaron secuencias T1, T2, STIR (short time inversion recovery) y DP con supresión grasa. Las imágenes fueron evaluadas por cuatro radiólogos con orientación en sistema musculoesquelético. Resultados: El 61.2\% de los pacientes presentaron RM normales, el 32.5\% presentaron alteraciones en la articulación sacroilíaca por imágenes y el 6.2\% fueron excluidos por presentar patologías diferentes a nuestra investigación. La lesión aguda se observó en el 76.9\% de los casos. La sacroileítis activa más cambios estructurales se observó en el 53.8\%. Conclusión: El uso de la RM como biomarcador para la actividad de la sacroileítis es el avance más actual, permite diagnosticar las etapas iniciales, antes que ocurra un daño estructural en el paciente.

Palabras clave: Sacroileítis. Criterios ASAS. Resonancia magnética.

Correspondencia:

*Silvia L. Espinoza-Alvarado

E-mail: s.lissespinoza@gmail.com

1665-2118/2019 Sociedad Mexicana de Radiologia e Imagen, AC. Publicado por Permanyer México SA de CV. Este es un artículo open access bajo la licencia CC BY-NC-ND (http://creativecommons.org/licenses/by-nc-nd/4.0/).
Recibido: 03-06-2019

Aceptado: 24-09-2019

DOI: $10.24875 / A R M .19000145$ 


\section{ABSTRACT}

Background: Magnetic resonance imaging (MRI) has become the most relevant imaging modality for the diagnosis and classification of early axial spondyloarthritis (SP), including early ankylosing spondylitis. Objectives: To determine the prevalence of sacroiliitis, identify and describe the MRI findings using the ASAS classification criteria (Assessment of SpondyloArthritis International Society). Method: Total of 80 patients with clinical suspicion of sacroiliitis and indication of sacroiliac MRI. They underwent MRI without gadolinium in a 1.5 Tesla resonator. T1, T2, STIR and DP sequences were performed with fat suppression. The images were evaluated by four radiologists with orientation in musculoskeletal system. Results: $61.2 \%$ of the patients presented normal MRI. $32.5 \%$ of the patients presented alterations in the sacroiliac joint by images, while $6.2 \%$ were excluded due to presenting different pathologies to our investigation. Acute injuries were observed in $76.9 \%$ of the cases. Active sacroileítis plus structural changes was observed $53.8 \%$. Conclusion: The use of MRI as a biomarker for the activity of sacroiliitis is the most current advance, it allows to diagnose the initial stages before structural damage occurs in the patient.

Key words: Sacroiliitis. ASAS criteria. Magnetic resonance.

\section{INTRODUCCIÓN}

La sacroileítis es un proceso inflamatorio que afecta las articulaciones sacroíliacas. Es un hallazgo distintivo y el más precoz en el diagnóstico del espectro de la espondiloartritis (SpA) debido a la alta frecuencia con que se afectan estas articulaciones. El concepto de SpA comprende espondilitis anquilosante, artritis psoriásica, artritis/espondilitis con enfermedad inflamatoria intestinal y artritis reactiva ${ }^{1}$.

Este grupo heterogéneo de enfermedades inflamatorias reumáticas crónicas comparten características clínicas, genéticas y fisiopatológicas comunes, así como asociación con el complejo principal de histocompatibilidad clase I antígeno leucocitario humano B27 (HLA-B27) ${ }^{2,3}$.

Dependiendo de la manifestación principal, se definen dos grupos de SpA principales: el concepto de SpA axial con afectación predominante de las articulaciones sacroilíacas y/o columna vertebral, y la SpA periférica no radiográfica con manifestaciones periféricas predominantes, tales como artritis, entesitis y dactilitis ${ }^{4}$.

Para una mejor comprensión, definición y unificación del concepto de enfermedad, la Assessment of SpondyloArthritis Internacional Society (ASAS) desarrolló nuevos criterios de clasificación para el reconocimiento temprano de la SpA. Según la ASAS, el concepto de SpA axial se define como la presencia de sacroileítis por radiografía o imágenes de resonancia magnética $(\mathrm{RM})^{5-7}$.

La RM es la innovación más reciente y el cambio importante con respecto a los criterios de clasificación establecidos anteriormente. Por primera vez, estos criterios incluyen el 
uso de imágenes de RM para el diagnóstico temprano y la medición de resultados objetivos para ensayos clínicos. Esta modalidad se ha convertido en una parte integral del manejo de pacientes con sacroileítis. Los criterios ASAS se aplican a los pacientes con al menos un historial de 3 meses de dolor de espalda que tienen menos de 45 años de edad al inicio del dolor (Tabla 1).

Para cumplir con la sección radiológica ha de demostrarse una sacroileítis mediante radiografía simple o $\mathrm{RM}^{8}$.

El objetivo de este estudio es determinar la prevalencia de sacroileítis, identificar y describir los hallazgos de RM utilizando los criterios de clasificación ASAS.

\section{MATERIAL Y MÉTODOS}

Estudio transversal, descriptivo con universo y muestra de 80 pacientes clínicamente sintomáticos, con dolor de espalda y sospecha clínica de sacroileítis e indicación de RM sacroilíaca. Todos los pacientes fueron examinados utilizando el siguiente protocolo de examen en un escáner de RM de 1,5 T (Philips Ingenia, Ámsterdam, Países Bajos), utilizando una bobina de columna vertebral y de cuerpo. Se realizaron corte axiales y coronales en secuencias T1, T2, STIR (short time inversion recovery) con cobertura bilateral de articulaciones sacroilíacas. Las imágenes fueron evaluadas por cuatro radiólogos expertos en el sistema musculoesquelético, y registraron las lesiones agudas y los cambios estructurales ocasionados por inflamación previa en la articulación sacroilíaca según los criterios de clasificación ASAS.

\begin{tabular}{l} 
TABLA 1. Características del dolor de espalda inflamatorio \\
Edad de inicio < 45 años \\
\hline Duración > 3 meses \\
Inicio insidioso \\
Rigidez matutina > 30 min \\
No mejora con el descanso \\
Despertar del dolor, especialmente durante la segunda mitad de \\
la noche, con una mejora al levantarse \\
Dolor alterno de glúteos \\
La presencia de dos o más de estas características debe despertar sospechas \\
de dolor de espalda inflamatorio, y la presencia de cuatro o más caracteristi- \\
cas puede considerarse diagnóstica. \\
La sensibilidad del dolor de espalda inflamatorio para el diagnóstico de la \\
espondiloartritis axial es del 70 a $80 \%$. La especificidad varía según la \\
población estudiada. \\
Fuente: Taurog J, et al. ${ }^{11}$
\end{tabular}

\section{Criterios de inclusión}

Pacientes mayores de 18 y menores de 45 años con sospecha clínica de sacroileítis e indicación de RM, y pacientes mayores de 45 años con inicio temprano de los síntomas (edad $<45$ años).

\section{Criterios de exclusión}

Pacientes posquirúrgicos, mayores de 45 años con síntomas recientes.

\section{Recolección de datos}

Se usó un instrumento que contenía información clínica y epidemiológica, que se aplicó al ingreso del paciente, y un instrumento para el análisis de las imágenes de RM.

\section{Procesamiento y análisis de datos}

Base de datos creada en el programa Epi info versión 4.1 del Centers for Disease Control and 
Prevention (CDC) de Atlanta. Se aplicaron las siguientes pruebas estadísticas: estadística descriptiva como frecuencia, porcentaje, media, mediana e intervalos de confianza al 95\%.

\section{Aspectos éticos}

Fue aprobado por el Comité de Ética de la Fundación Villavicencio.

\section{Definición de variables}

Edad y síntomas: Historial de 3 meses de dolor lumbar bajo, con una edad menor de 45 años al inicio del dolor (Tabla 1) 9-11. $^{9}$.

Las lesiones agudas: Edema óseo y osteítis, sinovitis, entesitis y capsulitis ${ }^{12}$.

Método de evaluación del edema medular óseo: Para cada articulación se evaluaron los cambios en cuatro localizaciones óseas: los huesos ilíaco y sacro correspondientes a las porciones cartilaginosa y ligamentosa de la articulación. La extensión del edema se dividió en 4 grados, donde 0 era normal (sin edema de médula ósea); 1 , leve ( $25 \%$ del área del hueso subcortical); 2 , moderado ( $25 \%$ a $50 \%$ del área ósea subcortical); y 3, grave (50\% o más del área ósea subcortical). Si las imágenes STIR mostraban una médula ósea brillante homogénea y si había alguna duda sobre si o no los cambios fueron causados por edema, se analizaron las imágenes ponderadas en T1. Las áreas con edema deben aparecer oscuras en esta secuencia ${ }^{13}$.

Los cambios crónicos, como la esclerosis subcondral y las erosiones óseas se evaluaron si estaban ausentes o presentes en al menos una región de la articulación sacroilíaca, tomando como modelo base un artículo publicado en el 2016 por la Arthritis \& Rheumatology ${ }^{14}$.

La transformación grasa de la médula ósea se evaluó mediante un sistema establecido y publicado por la revista, American College of Rheumatology en el año 2010, dividiendo su extensión en cuatro grados ${ }^{13}$ :

- Grado 0: Sin cambios de señal.

- Grado 1: 25\% del área subcortical.

- Grado 2: 25-50\% del área subcortical.

- Grado 3: 50\% del área subcortical.

La anquilosis se definió como puente óseo correspondiente al espacio articular ${ }^{13}$.

\section{ANATOMÍA}

La articulación sacroilíaca es la articulación axial más grande del cuerpo, con una superficie promedio de $17.5 \mathrm{~cm}^{2}$. Tiene una cápsula posterior ausente o rudimentaria ${ }^{15}$. Es una articulación compleja, con dos partes: la parte sinovial y la parte ligamentaria. La parte sinovial se localiza en el tercio anterior e inferior de la articulación (Fig. 1), tiene márgenes lisos y paralelos. La parte ligamentaria se localiza en los dos tercios dorsales y superiores de la articulación y muestra márgenes irregulares $^{16-19}$. En el adulto, el cartílago de la superficie sacra puede llegar a medir $4 \mathrm{~mm}$ de espesor, pero no exceder 1-2 $\mathrm{mm}$ en la superficie ilíaca ${ }^{17}$.

\section{MÉTODOS DIAGNÓSTICOS EN SACROILEÍTIS}

Históricamente, las articulaciones sacroilíacas se han evaluado mediante radiografía, pero 
TABLA 2. Sacroileitis: criterios ASAS

\section{Hallazgos requeridos}

Se requiere la presencia de lesiones activas en las articulaciones sacroilíacas para cumplir el criterio de "RM positiva para sacroileítis" y aplicarse en la clasificación diagnóstica de la ASAS

El edema óseo (STIR) u osteítis (T1-SG-Gd) son muy sugestivos de espondiloartritis, siempre y cuando afecten a las áreas subcondral y periarticular de la médula ósea

La existencia aislada de otras lesiones inflamatorias activas (sinovitis, entesitis 0 capsulitis) sin edema óseo ni osté́tis asociada NO es suficiente para la definición de sacroileítis por RM

Las lesiones estructurales (depósitos de grasa, erosiones, esclerosis o anquilosis) probablemente reflejan inflamación previa; sin embargo, su presencia sin edema óseo ni osteítis NO es suficiente para el diagnóstico de sacroileítis

\section{Alteración de señal obligatoria}

Si existe una sola lesión activa (edema óseo/osteítis), la alteración de señal deberá estar presente en al menos 2 cortes consecutivos

Si existe más de una alteración de señal (edema óseo/ osteítis) en un corte, es suficiente un solo corte

ASAS: Assessment of Spondylo Arthritis Internacional Society; RM: resonancia magnética; STIR: short time inversion recovery.

Fuente: Rudwaleit $M$, et al. ${ }^{4}$, Banegas Illescas, et al. ${ }^{23}$ y Rudwaleit, et al..$^{24}$

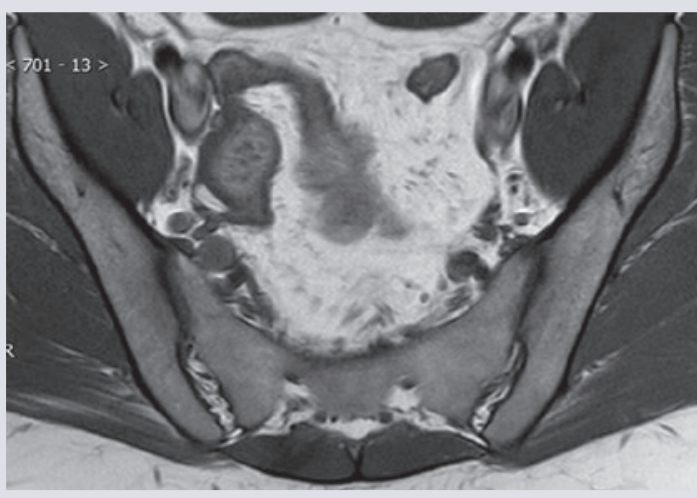

Figura 1. Anatomía normal. Secuencia T1, corte axial: la parte sinovial se localiza en el tercio anterior e inferior de la articulación y la parte ligamentaria se localiza en los dos tercios dorsales y superiores de la articulación.

solo puede mostrar cambios estructurales, como esclerosis, erosiones y anquilosis, y no puede detectar una inflamación temprana activa, lo que ocasiona un retraso en el diagnóstico. En la RM se pueden observar lesiones inflamatorias y estructurales mucho antes de que los cambios radiológicos sean evidentes ${ }^{20,21}$.

La tomografía computada (TC) es superior a la RM para visualizar la anatomía ósea detallada, los cambios estructurales patológicos, y puede mostrar cambios óseos crónicos en la sacroileítis, pero no signos de actividad inflamatoria. La TC emite radiación ionizante, por lo que no se usa como método diagnóstico en sacroileítis, especialmente en niños y adolescentes $^{22}$.

\section{Resonancia magnética}

En un enfoque consensual, la ASAS y el grupo Outcome Measures in Rheumatology Network, conformado por 8 reumatólogos y 2 radiólogos, considera la RM positiva para sacroileítis cuando se cumplen los siguientes criterios: lesiones inflamatorias activas (agudas) (edema óseo y osteítis, sinovitis, entesitis y capsulitis), pero solo el edema óseo y la osteítis son indispensables para el diagnóstico de sacroileítis activa (Tabla 2$)^{23}$.

Los nuevos criterios obtuvieron mejores resultados que las versiones modificadas del Grupo de Estudio de Esponlaritropatía Europea (ESSG) y los criterios de Amor. El uso de estos nuevos criterios en pacientes con dolor 
de espalda con o sin manifestaciones periféricas produjo un aumento en la sensibilidad $(79.5 \%)$ y especificidad (83.3\%) respecto a los criterios de ESSG y Amor. La prevalencia relativamente alta de sacroileítis en las imágenes (ya sea por radiografías o por RM) fue uno de los hallazgos más interesantes ${ }^{24}$.

En el protocolo de RM, para lograr una sensibilidad óptima a los cambios en la porción ligamentaria, se requieren imágenes en dos planos perpendiculares (coronal y axial oblicuo). Para la totalidad del hueso sacro debe tomarse una imagen desde su borde anterior hasta su borde posterior $\mathrm{y}$, generalmente, requiere al menos 10-12 secciones. El protocolo consiste en secuencias ponderadas en T1 oblicuas coronales y axiales para detectar cambios estructurales, y la secuencia STIR para detectar cambios inflamatorios agudos ${ }^{25}$.

\section{RESULTADOS}

Se evaluaron un total de 80 pacientes con dolor lumbar bajo de más de 3 meses de evolución, sospecha clínica de sacroileítis y solicitud de RM sin gadolinio. El $61.2 \%$ de los pacientes $(49 / 80)$ presentaron una RM normal. Los pacientes con alteraciones en la articulación sacroíliaca en las imágenes de RM fueron el 32.5\% (26/80) de los pacientes, y el $6.2 \%(5 / 80)$ fueron excluidos por presentar patologías diferentes a nuestra investigación.

Los pacientes menores de 45 años fueron $12 / 26$ y los mayores de 45 años con inicio temprano de dolor fueron 14/26. Los varones menores de 45 años fueron el 58.3\% (7/12) y los mayores de 45 años con inicio temprano de síntomas fueron el 41.6\% (5/12).
Las mujeres menores de 45 años fueron el $35.7 \%(5 / 14)$ y las mujeres mayores de 45 años con inicio temprano de síntomas, el $64.2 \%$ $(9 / 14)$. En cuanto a la prevalencia de patología por sexo, el 53.8\% (14/26) fueron mujeres y el $46.2 \% \%(12 / 26)$ fueron hombres.

Las lesiones agudas se observaron en el 76.9\% $(20 / 26)$. Los varones con lesión aguda fueron el $83.3 \%(10 / 12)$. El edema óseo se observó en el $83.3 \%(10 / 12)$ y entesitis, en el $8.3 \%$ $(1 / 12)$. La capsulitis y la osteítis no se evaluaron en nuestro estudio, porque no utilizamos sustancias paramagnéticas en nuestra investigación para su diagnóstico. Se observó derrame articular en el 8.3\% (1/12). En el sexo femenino se encontraron lesiones agudas en el $71.4 \%(10 / 14)$. El edema óseo se observó en el $90 \%(9 / 10)$ y el derrame articular, en el 10\% (1/10).

Los pacientes con sacroileítis activa más cambios estructurales en la articulación fueron el $53.8 \%(14 / 26)$. Los varones representaron el $50 \%(7 / 14)$ y las mujeres el $50 \%(7 / 14)$. Las mujeres mayores de 45 años con edema óseo más cambios estructurales fueron el $50 \%$ (7/14). Las mujeres menores de 45 años con edema más cambios estructurales fueron el $0 \%$. Los varones mayores de 45 años con edema más lesiones crónicas fueron el 16.6\% $(2 / 12)$ y los varones menores de 45 con edema más cambios estructurales representaron el $33.3 \%(4 / 12)$.

Las lesiones crónicas sin el biomarcador para sacroileítis activa se observaron en el $23.1 \%$ (6/26). En el sexo masculino se observaron en el 16.6\% (2/12) y el 100\% era mayor de 45 años, y en el sexo femenino representaron un $28.6 \%$ $(4 / 14)$ y el $100 \%$ era menor de 45 años. Las 
lesiones más frecuentes fueron la esclerosis subcondral en el 33.3\% (2/6), las erosiones en el 33.3\% (2/6), y los puentes óseos y anquilosis ósea en el $33.3 \%(2 / 6)$.

De los pacientes mayores de 45 años (14/26), el $64.2 \%$ (9/14) presentaron lesiones crónicas y el $100 \%$ pertenecía al sexo femenino. De los varones, el $80.0 \%(4 / 5)$ presentaron lesiones estructurales y el $20 \%(1 / 5)$ presentaron edema óseo.

La población mayor de 45 años presentó puentes óseos y anquilosis ósea en el 14.2\% $(2 / 14)$. Varones el $20 \%(1 / 5)$, mujeres el $20 \%(1 / 5)$.

\section{DISCUSIÓN}

Las lesiones agudas se identificaron con mayor frecuencia en la población masculina menor de 45 años, y el edema óseo fue el más frecuente (sacroileítis activa), identificadas como áreas hiperintensas en las secuencias STIR y áreas hipointensas en las secuencias $\mathrm{T}^{26}$. El edema fue moderado (afectación del 25 al 50\% del área subcortical). La localización más frecuente fue la región posteroinferior de la articulación en la zona subcondral y periarticular de la porción cartilaginosa de la articulación, que afecta con mayor frecuencia al hueso ilíaco e involucra ambas articulaciones sacroíliacas. La literatura refiere que el edema de la médula ósea en la sacroileítis relacionada con espondiloartropatía seronegativa afecta predominantemente a la parte inferior y posterior de la articulación sacroilíaca (Fig. 2) ${ }^{27}$, y comienza en la tercera década de la vida, con una relación hombre a mujer de dos a uno para la SpA axial radiográfica ${ }^{19}$.

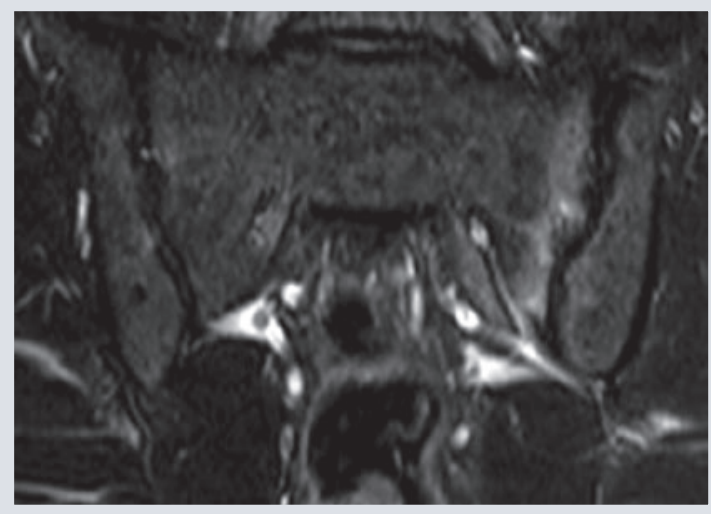

Figura 2. Edema óseo. Secuencia STIR, corte coronal: áreas de alta señal típicamente localizadas en la región periarticular y de predominio izquierdo.

Se observaron otras lesiones agudas en este rango de edad ( $<45$ años), pero nuestro estudio presentó limitantes en la evaluación de la sinovitis y la capsulitis debido a que se analizaron imágenes sin gadolinio. El uso de contraste intravenoso es necesario para la evaluación óptima de la sinovitis, la capsulitis y la tenosinovitis, pero no para la evaluación de las erosiones, la proliferación ósea y el edema de la médula ósea ${ }^{28}$.

Además, los criterios ASAS establecen que sin edema óseo no es suficiente para diagnosticar sacroileítis activa, y el diagnóstico de la SpA axial requiere la presencia de edema de médula ósea subcondral o periarticular en articulaciones sacroilíacas en STIR o secuencias ponderadas en T2 con saturación gra$\mathrm{sa}^{29,30}$. Por esta razón, en nuestro estudio nos limitamos a identificar únicamente derrame articular en la secuencia STIR.

La entesitis se observó en menor frecuencia, los hallazgos se describen en la literatura como una señal hiperintensa en imágenes STIR y T1 más gadolinio en el sitio de 
inserción de tendones y ligamentos, y la alteración de señal puede extenderse a la médula ósea y al tejido blando ${ }^{29}$. En este estudio no utilizamos gadolinio, pero en la secuencia STIR se identificó la hiperintensidad en la inserción de los ligamentos.

La población mayor de 45 años, pero con inicio temprano de síntomas, fue predominante en el sexo femenino, y al evaluar las imágenes de RM se observó el edema óseo (biomarcador principal para sacroileítis) establecido por la ASAS. Se observaron cambios estructurales, probablemente debidos a episodios agudos previos, y la primera en frecuencia fue la esclerosis subcondral. Estas lesiones se observaron como bandas de baja intensidad en las secuencias T1 y STIR. Su extensión fue mayor de $5 \mathrm{~mm}$, y la moda fue de $7.3 \mathrm{~mm}$. Según los criterios ASAS, la esclerosis atribuible a SpA debe extenderse al menos $5 \mathrm{~mm}$ desde el espacio de la articulación sacroilíaca y no muestra realce después de la administración del medio de contraste (Fig. 3 y Tabla 3) 30,31. La segunda lesión crónica más frecuente fueron las erosiones óseas. Se evaluaron en la secuencia T1, identificándolas de forma predominante en el aspecto anteroinferior de la articulación sacroilíaca (Fig. 4), y el área subcondral del hueso ilíaco fue la más afectada. Según la literatura internacional se afecta con más frecuencia al hueso ilíaco que el sacro. Se identifican como defectos óseos en los márgenes articulares que al confluir producen un pseudoensanchamiento del espacio articular (Tabla 3) 32 .

Los puentes óseos y anquilosis se identificaron en la población mayor de 45 años, con afectación de ambos sexos en igual porcentaje.

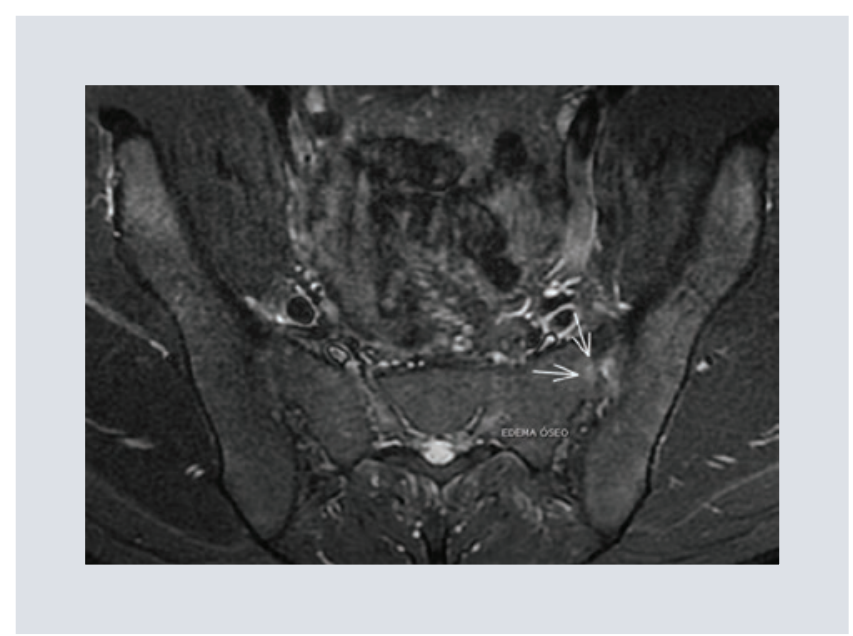

Figura 3. Secuencia STIR, corte coronal, edema óseo bilateral periarticular de predominio izquierdo (flechas).

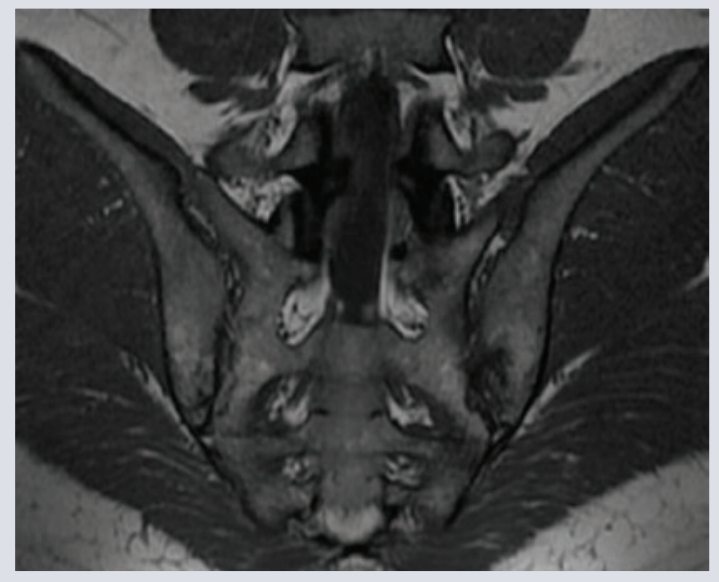

Figura 4. Erosiones óseas bilaterales. Secuencia T1, corte axial: defectos óseos confluentes localizados en los márgenes articulares en la región anteroinferior de la articulación sacroilíaca. Producen pseudoensanchamiento del espacio articular izquierdo.

Se visualizaron como áreas de baja señal en todas las secuencias (Figs. 5 y 6). En estados avanzados generan pérdida de la definición del espacio articular y fusión ósea (Tabla 3) ${ }^{33,34}$. La articulación puede desaparecer completamente en la enfermedad tardía con anquilosis y remodelación del hueso ${ }^{35}$. En nuestro estudio se identificó únicamente un paciente con fusión ósea asociada con áreas de edema 
TABLA 3. Criterios ASAS

\begin{tabular}{|l|l|l|}
\hline \multicolumn{2}{ll}{ Cambios estructurales en sacroileítis } \\
\hline Lesiones cróncias & Secuencias & Hallazgos \\
\hline Esclerosis subcondral & T1 y STIR & Bandas de baja intensidad. Extensión > de 5 mm desde el espacio de la articulación \\
\hline Erosiones óseas & T1: hipointensos & Defectos óseos en el área subcondral del hueso ilíaco y sacro \\
\hline Erosiones óseas activas & STIR: hiperintensos & Defectos óseos en el área subcondral del hueso ilíaco y sacro \\
\hline Transformación grasa & T1 & Señal hiperintensa en la región subcortical de la articulación sacroilíaca \\
\hline Puentes óseos & T1 y STIR & Áreas de baja señal en el espacio articular \\
\hline Anquilosis ósea & T1 y STIR & Pérdida de la definición del espacio articular y fusión ósea \\
\hline
\end{tabular}

ASAS: Assessment of SpondyloArthritis Internacional Society, RM: resonancia magnética; STIR: short time inversion recovery.

Fuente: Rudwaleit, et al. ${ }^{24}$, Maksymowych, et al. ${ }^{44}$ y Maksymowych, et al. ${ }^{45}$

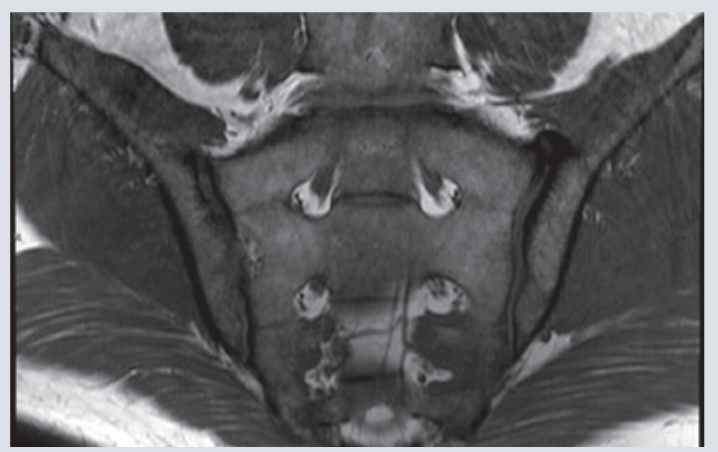

Figura 5. Puentes óseos. Secuencia T, corte coronal: áreas de baja señal en la articulación sacroilíaca izquierda, formando un puente que atraviesa la articulación.

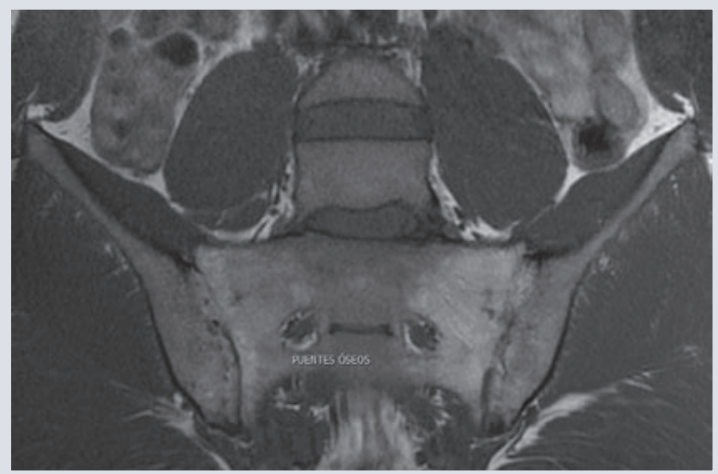

Figura 6. Anquilosis ósea. Secuencia T1, corte coronal: pérdida de la definición del espacio articular y fusión ósea bilateral. subarticular. Según estudios publicados, este hallazgo sugiere que la fusión no indica necesariamente la ausencia de actividad inflamatoria en la articulación sacroilíaca ${ }^{36}$.

El depósito de médula ósea grasa periarticular se identificó con mayor frecuencia en la población mayor de 45 años. Se observan como áreas de mayor señal en secuencias ponderadas en T1. Según la literatura no es un hallazgo específico y probablemente representa zonas de inflamación reparada y se considera como un evento postinflamatorio, además de ser un importante tejido intermediario para la formación de hueso nuevo en la articulación sacroilíaca, y se sugiere que la terapia debe dirigirse a una etapa temprana de la enfermedad antes de que comiencen los procesos de transformación de la grasa y la formación de hueso nuevo ${ }^{37-40}$.

La población femenina también presentó características llamativas. Su edad diagnóstica es mayor de los 45 años, superando lo establecido por los criterios ASAS, y se observa una moda de 55 años de edad y cambios 
estructurales en las articulaciones sacroilíacas sugestivos de eventos inflamatorios previos. Sin embargo, se ha informado una demora sustancial entre la aparición de los síntomas y el diagnóstico posterior por parte de un reumatólogo. Una explicación importante para el retraso en la derivación en la SpA axial es que el síntoma principal de la SpA axial, es muy común, especialmente en la atención primaria, dado que los médicos de atención primaria y otros especialistas referentes pueden no estar suficientemente conscientes de los signos específicos de la enfermedad $^{41-43}$.

Un pequeño porcentaje de pacientes (4\%) presentó entesitis bilateral acompañado de lesiones estructurales y derrame articular sin presencia de edema óseo, lo cual según los criterios ASAS no es suficiente para establecer el diagnóstico de sacroileítis activa ${ }^{9}$.

El 23\% de los pacientes reflejaron inflamación previa, y se observaron lesiones estructurales sin presencia de edema óseo, por lo que no se englobaron en el término de sacroileítis.

El 8\% de los pacientes tenían el diagnóstico de sacroileítis y se solicitaba una RM de control. Estos pacientes no presentaron estudios previos para evaluar la mejoría con el tratamiento. Estos pacientes se tomaron como un proceso inicial debido a que los criterios ASAS no toman en cuenta si el paciente es recidivante.

\section{CONCLUSIONES}

1. La sacroileítis activa se observó con mayor frecuencia en la población masculina menor de 45 años, la mayoría de los pacientes presentó un edema grado II (moderado) con mayor afectación de la porción cartilaginosa de la articulación y que involucraba ambas articulaciones sacroilíacas.

2. La sacroileítis activa con lesiones estructurales fue más frecuente en pacientes del sexo femenino mayores de 45 años, y las lesiones más frecuentes fueron la esclerosis ósea, erosiones y los puentes óseos.

3. Las lesiones crónicas sin el biomarcador para sacroileítis activa predominaron en mujeres menores de 45 años.

4. El uso de la RM como un biomarcador para la actividad de la enfermedad y como una guía para el tratamiento de la sacroileítis es el avance más actual, y permite diagnosticar y tratar las etapas iniciales, antes que ocurra un daño estructural en el paciente.

\section{RECOMENDACIONES}

Las normas ASAS se han desarrollado para ayudar a la derivación y el diagnóstico temprano de pacientes con una alta sospecha de AxSpA a reumatólogos, pero de acuerdo con otros autores se debe establecer un método de lectura para la RM control, ya que los criterios no lo toman en cuenta.

\section{AGRADECIMIENTOS}

Roberto Villavicencio: Director Médico de la Fundación Villavicencio.

Claudio Bonini: Director Médico de Diagnóstico Médico Oroño. 


\section{CONFLICTO DE INTERESES}

Los autores declaran no tener conflictos de intereses.

\section{FINANCIAMIENTO}

Los autores declaran que recibieron financiamiento de Diagnóstico Médico Oroño y apoyo con equipo de resonancia magnética.

\section{RESPONSABILIDADES ÉTICAS}

Protección de personas y animales. Los autores declaran que para esta investigación no se han realizado experimentos en seres humanos ni en animales.

Confidencialidad de los datos. Los autores declaran que han seguido los protocolos de su centro de trabajo sobre la publicación de datos de pacientes.

\section{Derecho a la privacidad y consentimiento} informado. Los autores han obtenido el consentimiento informado de los pacientes y/o sujetos referidos en el artículo. Este documento obra en poder del autor de correspondencia.

\section{BIBLIOGRAFÍA}

1. Rudwaleit M, van der Heijde D, Landewe R, Listing J, Akkoc N, Brandt J, et al. The development of Assessment of SpondyloArthritis international Society classification criteria for axial spondyloarthritis (part II): validation and final selection. Ann Rheum Dis. 2009;68(6):777-83.

2. Proft F, Poddubnyy D. Ankylosing spondylitis and axial spondyloarthritis: recent insights and impact of new classification criteria. Ther Adv Musculoskelet Dis. 2018;10(5-6):129-39.

3. Hammoudeh M, Abdulaziz S, Alosaimi H, Al-Rayes H, Aldeen Sarakbi H, Baamer $\mathrm{M}$ et al. Challenges of diagnosis and management of axial spondyloarthritis in North Africa and the Middle East: An expert consensus. J Inter Med Res. 2016;44(2):216-30.
4. Rudwaleit M, Landewe R, van der Heijde D, Listing J, Brandt J, Braun J, et al. The development of Assessment of SpondyloArthritis international Society classification criteria for axial spondyloarthritis (part I): classification of paper patients by expert opinion including uncertainty appraisal. Ann Rheum Dis. 2009;68(6):770-6.

5. Rueda J, Arias-Correal S, Vasquez A, Calvo E, Peña P, Porras M, et al. Interobserver Agreement in Magnetic Resonance of the Sacroiliac Joints in Patients with Spondyloarthritis. Int J Rheumatol. 2017; Article ID 3143069, 5 pages.

6. Min H, Lee J, Ju J, Park S, Kwok S. Predictors of Assessment of Spondyloarthritis International Society (ASAS) Health Index in Axial Spondyloarthritis and Comparison of ASAS Health Index between Ankylosing Spondylitis and Nonradiographic Axial Spondyloarthritis: Data from the Catholic Axial Spondyloarthritis COhort (CASCO). J Clin Med. 2019;8(4):467.

7. Raychaudhuri S, Deodhar A. The classification and diagnostic criteria of ankylosing spondylitis. J Autoimmun. 2014;48-49:128-33.

8. Navallas M, Ares J, Beltrán B, Lisbona M, Maymó J, Solano A. Sacroiliitis Associated with Axial Spondyloarthropathy: New Concepts and Latest Trends. Radio Graphics. 2013;33(4):933-56.

9. Arnbak B, Leboeuf-Yde C, Jensen T. A systematic critical review on MRI in spondyloarthritis. Arthritis Research \& Therapy. 2012;14(2):R55.DOI:

10. Jacobson J, Girish G, Jiang Y, Resnick D. Radiographic Evaluation of Arthritis: Inflammatory Conditions. Radiology. 2008;248(2):378-89.

11. Taurog J, Chhabra A, Colbert RA. Ankylosing Spondylitis and Axial Spondyloarthritis. N Engl J Med. 2016;374(26):2563-74.

12. Sudoł-Szopińska I, Kwiatkowska B, Włodkowska-Korytkowska M, Matuszewska G, Grochowska E. Diagnostics of Sacroiliitis According to ASAS Criteria: A Comparative Evaluation of Conventional Radiographs and MRI in Patients with a Clinical Suspicion of Spondyloarthropathy. Preliminary Results. Pol J Radiol. 2015;80:266-76.

13. Madsen K, Jurik A. Magnetic resonance imaging grading system for active and chronic spondylarthritis changes in the sacroiliac joint. Arthritis Care Res. 2010;62(1):11-8.

14. Arnbak B, Grethe Jurik A, Hørslev-Petersen K, Hendricks O, Hermansen L, Loft A, et al. Associations Between Spondyloarthritis Features and Magnetic Resonance Imaging Findings: A Cross-Sectional Analysis of 1,020 Patients With Persistent Low Back Pain. Arthritis Rheumatol. 2016; 68(4):892-900.

15. Cohen S. Sacroiliac Joint Pain: A Comprehensive Review of Anatomy, Diagnosis, and Treatment. Anesth Analg. 2005;101(5):1440-53.

16. Herregods N, Dehoorne J, Jaremko J, Joos R, Baraliakos X, Verstraete K, et al. Diagnostic Value of MRI of Sacroiliac Joints in Juvenile Spondyloarthritis. J Belg Soc Radiol. 2016;100(1):95.

17. Vleeming A, Schuenke M, Masi A, Carreiro J, Danneels L, Willard F. The sacroiliac joint: an overview of its anatomy, function and potential clinical implications. J Anatomy. 2012;221(6):537-67

18. Zaidi H, Montoure A, Dickman C. Surgical and clinical efficacy of sacroiliac joint fusion: a systematic review of the literature. Journal of Neurosurgery: Spine. 2015;23(1):59-66

19. Prabhu S, Irodi A, Prakash D. Seronegative spondyloarthropathy-related sacroiliitis: CT, MRI features and differentials. Indian J Radiol Imaging. 2014;24(3):271

20. Lai A, Sheu L, Gensler L, McQuaid K, Dhaliwal G. A Terminal Event. N Engl J Med. 2019;381(10):970-6

21. Mandl P, Navarro-Compán V, Terslev L, Aegerter P, van der Heijde D, $\mathrm{D}^{\prime}$ Agostino $\mathrm{M}$ et al. EULAR recommendations for the use of imaging in the diagnosis and management of spondyloarthritis in clinical practice. Ann Rheuma Dis. 2015;74(7):1327-39.

22. Zejden A, Jurik A. Anatomy of the sacroiliac joints in children and adolescents by computed tomography. Pediatric Rheumatol Online J. 2017;15:82.

23. Banegas Illescas M, López Menéndez C, Rozas Rodríguez M, Fernández Quintero R. Nuevos criterios ASAS para el diagnóstico de espondiloartritis. Diagnóstico de sacroileítis por resonancia magnética. Radiología. 2014 56(1):7-15. 
24. Rudwaleit M, van der Heijde D, Landewe R, Akkoc N, Brandt J, Chou C, et al. The Assessment of SpondyloArthritis international Society classification criteria for peripheral spondyloarthritis and for spondyloarthritis in general. Ann Rheum Dis. 2010;70(1):25-31.

25. Navallas M, Ares J, Beltrán B, Lisbona M, Maymó J, Solano A. Sacroiliitis Associated with Axial Spondyloarthropathy: New Concepts and Latest Trends. RadioGraphics. 2013;33(4):933-56.

26. Tarantino A, Jablonska J, D'Aprile P. All that glitters is not gold: sacroiliitis. Reumatologia/Rheumatology. 2018;56(5):289-93.

27. Sieper J, Poddubnyy D. Axial spondyloarthritis. Lancet. 2017;390:73-84.

28. Felbo S, Terslev L, Østergaard M. Imaging in peripheral and axial psoriatic arthritis: contributions to diagnosis, follow-up, prognosis and knowledge of pathogenesis. Clini Exp Rheumatology 2018. 2018;36(114):24-34.

29. Rudwaleit M, Jurik A, Hermann K, Landewe R, van der Heijde D, Baraliakos $\mathrm{X}$ et al. Defining active sacroiliitis on magnetic resonance imaging (MRI) for classification of axial spondyloarthritis: a consensual approach by the ASAS/OMERACT MRI group. Ann Rheum Dis. 2009;68(10):1520-7.

30. Weiss P, Xiao R, Biko D, Chauvin N. Assessment of Sacroiliitis at Diagnosis of Juvenile Spondyloarthritis by Radiography, Magnetic Resonance Imaging, and Clinical Examination. Arthritis Care Res. 2016;68(2):187-94.

31. Aydin S, Maksymowych W, Bennett A, McGonagle D, Emery P, MarzoOrtega H. Validation of the ASAS criteria and definition of a positive MRI of the sacroiliac joint in an inception cohort of axial spondyloarthritis followed up for 8 years. Annals of the Rheumatic Diseases. 2011;71(1):56-60.

32. Sieper J, Rudwaleit M, Baraliakos X, Brandt J, Braun J, Burgos-Vargas R, et al. The Assessment of SpondyloArthritis international Society (ASAS) handbook: a guide to assess spondyloarthritis. Ann Rheum Dis. 2009;68(Suppl2):ii1-ii44.

33. Sarmiento de la Iglesia MM, Diez Renovales F, Lecumberri Cortés G, Cardenal Urdampilleta J, Corta Gomez I, Grande Icaran D. Actualización en el diagnóstico por imagen de la sacroileítis. SERAM 2012; S-1057.

34. Weber U, Pedersen S, Ostergaard M, Rufibach K, Lambert R, Maksymowych W. Can erosions on MRI of the sacroiliac joints be reliably detected in patients with ankylosing spondylitis? A cross-sectional study. Arthr Res Ther. 2012;14(3):R124.
35. Schiappacasse FG, Díaz JJ, Alvayay QP. Protocolo abreviado de resonancia magnética en espondiloartritis: más allá de la sacroileítis. Rev Med Chile. 2015;143(7):905-12.

36. Østergaard M, Lambert R. Imaging in ankylosing spondylitis. Therap Adv Musculoskeletal Dis. 2012;4(4):301-11.

37. Bredella M, Steinbach L, Morgan S, Ward M, Davis J. MRI of the Sacroiliac Joints in Patients with Moderate to Severe Ankylosing Spondylitis. Am J Roentgenol. 2006;187(6):1420-6.

38. Maksymowych W, Wichuk S, Chiowchanwisawakit P, Lambert R, Pedersen S. Fat metaplasia on MRI of the sacroiliac joints increases the propensity for disease progression in the spine of patients with spondyloarthritis. RMD Open. 2017;3(1):e000399.

39. Kang K, Kim I, Yoon M, Hong Y, Park S, Ju J. Fat Metaplasia on Sacroiliac Joint Magnetic Resonance Imaging at Baseline Is Associated with Spinal Radiographic Progression in Patients with Axial Spondyloarthritis. PLoS One. 2015;10(8):e0135206.

40. Marzo-Ortega H, Gaffney K, Gaffney K. Defining the target: clinical aims in axial spondyloarthritis. Rheumatology. 2018;57(Suppl 6): vi18-vi22.

41. Deodhar A, Mease P, Reveille J, Curtis J, Chen S, Malhotra K, et al. Frequency of Axial Spondyloarthritis Diagnosis Among Patients Seen by US Rheumatologists for Evaluation of Chronic Back Pain. Arthritis Rheumatol. 2016;68(7):1669-76

42. Rudwaleit M, Sieper J. Referral strategies for early diagnosis of axial spondyloarthritis. Nat Rev Rheumatol. 2012;8(5):262-8.

43. Abawi O, van den Berg R, van der Heijde D, van Gaalen F. Evaluation of multiple referral strategies for axial spondyloarthritis in the SPondyloArthritis Caught Early (SPACE) cohort. RMD Open. 2017;3(1):e000389

44. Maksymowych W, Wichuk S, Dougados M, Jones H, Szumski A, Bukowski J, et al. MRI evidence of structural changes in the sacroiliac joints of patients with non-radiographic axial spondyloarthritis even in the absence of MRI inflammation. Arthritis Res Ther. 2017;19(1):126.

45. Maksymowych W, Wichuk S, Chiowchanwisawakit P, Lambert R, Pedersen S. Fat Metaplasia and Backfill Are Key Intermediaries in the Development Sacroiliac Joint Ankylosis in Patients With Ankylosing Spondylitis. Arthritis Rheumatol. 2014;66(11):2958-67. 